\title{
EFEITO DA DISPONIBILIDADE HÍDRICA E DA APLICAÇÃO DE POTÁSSIO E SÓDIO NAS CARACTERÍSTICAS ANATÔMICAS DO LENHO JUVENIL DE Eucalyptus grandis ${ }^{1}$
}

Paola de Castro e Freitas ${ }^{2}$, Carlos Roberto Sette $\mathrm{Jr}^{3}$, Vinicius Resende de Castro ${ }^{4}$, Gilles Chaix ${ }^{5}$, Jean Paul Laclau ${ }^{5}$ e Mario Tomazello Filho ${ }^{6}$

\begin{abstract}
RESUMO - A disponibilidade de água e de fertilizantes em povoamentos florestais promove estímulos na atividade do meristema cambial das árvores, causando significativas alterações nas características anatômicas e na formação do lenho. O estudo teve como objetivo avaliar as características anatômicas do lenho de árvores de Eucalyptus grandis com 24 e 36 meses de idade submetidas à redução das chuvas e à nutrição de $\mathrm{K}$ e Na. Os tratamentos foram definidos por dois regimes hídricos (100\% e 66\% das chuvas, com redução artificial com lonas de polietileno) e três tipos de nutrição: $\mathrm{K}(4,5 \mathrm{kmol} / \mathrm{ha}), \mathrm{Na}(4,5 \mathrm{kmol} / \mathrm{ha})$ e controle. Foram selecionadas três árvores por tratamento e por idade, totalizando 36 árvores amostradas, sendo seccionados Discos de lenho a 1,3 m de altura (DAP) para a avaliação das características anatômicas. Os resultados indicaram que os tratamentos de nutrição $\mathrm{x}$ disponibilidade hídrica induziram alterações na largura total, diâmetro do lume e espessura da parede da fibra aos 24 meses e não alteraram as dimensões dos vasos no lenho do E. grandis. A aplicação isolada do K e do Na não influenciou as características anatômicas do lenho, exceto para a largura, diâmetro do lume e espessura da parede da fibra aos 24 meses. A redução dos níveis de precipitação, pela instalação de faixas de lonas de polietileno, não influenciou significativamente as dimensões das fibras e dos vasos no lenho das árvores de eucalipto. Os resultados poderão ser utilizados como subsídios para a adoção de práticas silviculturais nas plantações florestais em áreas com estresse hídrico, bem como a substituição parcial de K por Na.
\end{abstract}

Palavras-chave: Nutrição; Estresse hídrico induzido; Formação do lenho.

\section{EFFECT OF WATER AVAILABILITY AND APPLICATION OF POTASSIUM AND SODIUM IN THE JUVENILE WOOD ANATOMICAL CHARACTERISTICS OF Eucalyptus grandis}

\begin{abstract}
The availability of water and fertilizer in forest stands promotes changes in cambial activity of trees, causing significant changes in wood anatomical features and wood formation. The study aimed to evaluate the wood anatomical characteristics of Eucalyptus grandis trees at 24 and 36 months with reduced rainfall and nutrition of $K$ and $N a$. Treatments were defined by two water regimes $(100 \%$ and $66 \%$ of rainfall, artificial reduction with polyethylene tarps) and three types of nutrition: $K(4,5 \mathrm{kmol} / \mathrm{ha}), \mathrm{Na}(4,5 \mathrm{kmol} / \mathrm{ha})$ and control. 3 eucalyptus trees were selected per treatment and age, totaling 36 sampled trees, and discs samples of wood at $1.3 \mathrm{~m}$ height $(\mathrm{DBH})$ were evaluated in relation to anatomical characteristics. The results showed that treatments nutrition $x$ water availability induced changes in width, wall thickness and lumen diameter of the fiber at 24 months and did not alter the vessel dimensions in wood of E. grandis trees. The
\end{abstract}

\footnotetext{
${ }^{1}$ Recebido em 24.08.2014 aceito para publicação em 02.02.2015.

${ }^{2}$ Universidade Federal de Goiás, Programa de Pós-graduação em Agronomia, Goiânia, GO-Brasil.E-mail: <paolacastrofreitas@gmail.com>. ${ }^{3}$ Universidade Federal de Goiás, Departamento de Engenharia Florestal, Goiânia, GO - Brasil. E-mail: <crsettejr@hotmail.com>.

${ }^{4}$ Universidade de São Paulo, Escola Superior de Agricultura Luiz de Queiroz, Programa de Pós-graduação em Recursos Florestais, Piracicaba, SP - Brasil. E-mail: <vresende@gmail.com>.

${ }^{5}$ Centre de Recherche Agronomique Pour le Développement, Montpellier - France. E-mail: <gilles.chaix@cirad.fr> e $<$ jean-paul.laclau@cirad.fr>.

${ }^{6}$ Universidade de São Paulo, Escola Superior de Agricultura Luiz de Queiroz, Departamento de Ciências Florestais, Piracicaba, SP - Brasil. E-mail: <mtomazel@usp.br>.
} 
effect of isolated application of $K$ and Na does not influence the wood anatomical characteristics of $\mathbf{E}$. grandis, except for width, lumen diameter and wall thickness at 24 months. The reduction of the rainfall, with installation of polyethylene tarp, does not significantly influence the dimension of the fibers and the vessels of wood in the eucalyptus trees. The results may be used as subsidies for the adoption of silvicultural practices in forest plantations in areas with water stress, as well as the partial replacement of $K$ by Na.

Keywords: Nutrition; Induced water stress; Wood formation.

\section{INTRODUÇÃO}

No Brasil, a maioria das áreas com cultivo de espécies do gênero Eucalyptus são estabelecidas em regiões de baixa fertilidade, particularmente pobres em potássio, e submetidas a períodos de déficit hídrico (GAVA, 1997). A compreensão dos processos e das interações entre a disponibilidade potássica e hídrica sobre o funcionamento dos povoamentos de eucalipto, especialmente nas características anatômicas do lenho das árvores, torna-se determinante, uma vez que os mecanismos do potássio estão diretamente relacionados com o balanço hídrico das árvores. Isso faz aumentar a eficiência do uso da água sob estresse hídrico, promovendo maior resistência das árvores à seca, influenciando a atividade cambial e formação do lenho (ALMEIDA, 2009).

Diante dos cenários atuais e futuros dos efeitos das mudanças climáticas globais que preveem alterações nos regimes hídricos em várias regiões do Brasil (INTERGOVERNMENTAL PANEL ON CLIMATE CHANGE - IPCC, 2007; NOBRE et al., 2007), as florestas naturais e os plantios florestais podem ser diretamente afetados. $O$ estresse hídrico tem provocado a diminuição do crescimento e o aumento da mortalidade das árvores (CABRAL et al., 2010). Os estímulos provocados na atividade cambial das plantas em decorrência da disponibilidade hídrica do ambiente e da aplicação de potássio e do sódio promovem significativas alterações nas suas características anatômicas e no processo de formação do lenho (DREW, M. et al., 2009; SETTE JR. et al., 2010), sendo fundamental o seu estudo. Várias pesquisas têm sido realizadas em ambiente controlado para avaliar os efeitos do estresse hídrico sobre o crescimento inicial de diferentes espécies de eucalipto (COOPMAN et al., 2008). Contudo, os estudos sobre os efeitos de um estresse hídrico severo e prolongado no campo, nas características anatômicas do lenho de árvores de eucalipto, são escassos.
Os minerais são essenciais em muitos processos fisiológicos das plantas, e a nutrição é conhecida como importante fator que controla o seu crescimento e desenvolvimento (GONÇALVES; BENEDETTI, 2000). No entanto, o conhecimento das funções dos minerais nas árvores ainda é insatisfatório e restrito aos efeitos no incremento em diâmetro e altura do tronco. Nos últimos anos, as pesquisas têm revelado correlação positiva entre o potássio e a atividade cambial das árvores (WIND et al., 2004; FROMM, 2010). Apesar de os estudos iniciais revelarem alguma influência dos minerais na atividade cambial e formação do lenho, existe ainda a necessidade de informações mais detalhadas sobre o envolvimento específico desses elementos, especialmente do sódio, durante o processo de crescimento cambial e consequente formação do lenho.

Alguns estudos apontaram que a aplicação do potássio e do sódio altera a sua concentração na região cambial (SETTE JR. et al., 2013), promovendo aumento no potencial osmótico, acarretando na expansão celular e, consequentemente, aumentando a atividade cambial e alterações nas características anatômicas do lenho. Entretanto, poucos trabalhos têm sido desenvolvidos para avaliar o efeito do sódio no crescimento e qualidade da madeira em plantações florestais de eucalipto (SETTE JR. et al., 2014).

Diante do exposto, este trabalho tem como objetivo avaliar o efeito da disponibilidade de água e da aplicação de potássio e sódio nas características anatômicas do lenho juvenil de árvores de Eucalyptus grandis.

\section{MATERIAL E MÉTODOS}

\subsection{Caracterização da área de estudo}

O experimento foi instalado na Estação Experimental de Ciências Florestais de Itatinga, SP, localizada a $23^{\circ}$ $10^{\prime} \mathrm{S}$ e $2348^{\circ} 40^{\prime} \mathrm{W}$, com $857 \mathrm{~m}$ de altitude, por meio do convênio do Departamento de Ciências Florestais

Revista Árvore, Viçosa-MG, v.39, n.2, p.405-416, 2015

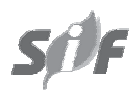


da ESALQ/USP e do Centro de Cooperação Internacional em Pesquisa Agronômica para o Desenvolvimento (CIRAD)/França. O experimento faz parte de amplo grupo de pesquisas que compõem o Projeto Universal CNPq n’ 471808/2012-1 "Mudanças climáticas globais: indução do estresse hídrico por redução de chuva e efeito no crescimento e formação e qualidade do lenho de árvores de Eucalyptus grandis com nutrição potássica e sódica".

O clima da região é caracterizado como mesotérmico úmido (Cwa), segundo Köeppen, com precipitação média anual de $1.400 \mathrm{~mm}$ e temperatura média de $16,2{ }^{\circ} \mathrm{C}$ e $28,6{ }^{\circ} \mathrm{C}$ nos meses mais frios e mais quentes, respectivamente. O solo é do tipo Latossolo VermelhoAmarelo distrófico de textura média (200 g kg-1 de argila) com a litologia composta por arenito, formação Marília, do Grupo Bauru.

\subsection{Delineamento experimental}

O ensaio foi implantado em delineamento experimental em blocos subdivididos tipo "splitplot" com três blocos e seis tratamentos, totalizando 18 parcelas. Os tratamentos foram definidos por dois regimes hídricos, $100 \%$ das chuvas e $66 \%$ das chuvas, com exclusão artificial por $1.700 \mathrm{~m}^{2}$ de lonas em polietileno (Figura 1$)$ e três doses de fertilizantes $(0=$ controle; $4,5 \mathrm{kmol} /$ ha e $4,5 \mathrm{kmol} / \mathrm{ha}$ na forma de $\mathrm{KCl}$ e $\mathrm{NaCl}$, respectivamente, aplicados três meses após o plantio).

Os tratamentos foram identificados da seguinte forma: (a)C/+C, controle $100 \%$ de chuva; (b)Na/
+C, sódio $100 \%$ de chuva; (c) $\mathbf{K} /+\mathbf{C}$, potássio $100 \%$ de chuva; (d)C/-C, controle $66 \%$ de chuva; (e)Na/ -C, sódio $66 \%$ de chuva; e (f) $\mathbf{K} /$-C, potássio $66 \%$ de chuva. O plantio foi realizado no espaçamento de $3 \times 2 \mathrm{~m}$, sendo, antes do plantio em todos os tratamentos, distribuídos a lanço e sem incorporação, $2.000 \mathrm{~kg}$ de calcário dolomítico por hectare. A adubação de plantio foi feita nas covas, fornecendo-se o equivalente a 80 $\mathrm{kg}$ de $\mathrm{P}_{2} \mathrm{O}_{5}$ (Super Fosfato Simples), $30 \mathrm{~kg}$ de N $\left(\mathrm{NH}_{4}\left(\mathrm{SO}_{4}\right)_{2}\right)$ e $30 \mathrm{~kg}$ de FTE (BR-12) por hectare, como fonte de micronutrientes.

\subsection{Características anatômicas do lenho}

Para a análise das dimensões das fibras e dos vasos, inicialmente foram selecionadas três árvores por idade e tratamento ( 2 idades x 6 tratamentos x 3 árvores; total de 36 árvores amostradas), representativas da classe de área basal média do plantio; e cortados discos do lenho do DAP, bem como feita a posterior retirada de corpos de prova, em três posições radiais $(0,50$ e 100\% do raio).

Para avaliação das fibras, foram retiradas amostras dos corpos de prova nas três posições radiais e submetidas ao processo de maceração, segundo o método Franklin (JOHANSEN, 1940). As lâminas histológicas com as fibras foram analisadas em microscopia de luz e coletadas 40 imagens de fibras por lâmina, para a mensuração do comprimento (ampliação de 40 vezes), largura e diâmetro do lume (ampliação de 400 vezes).

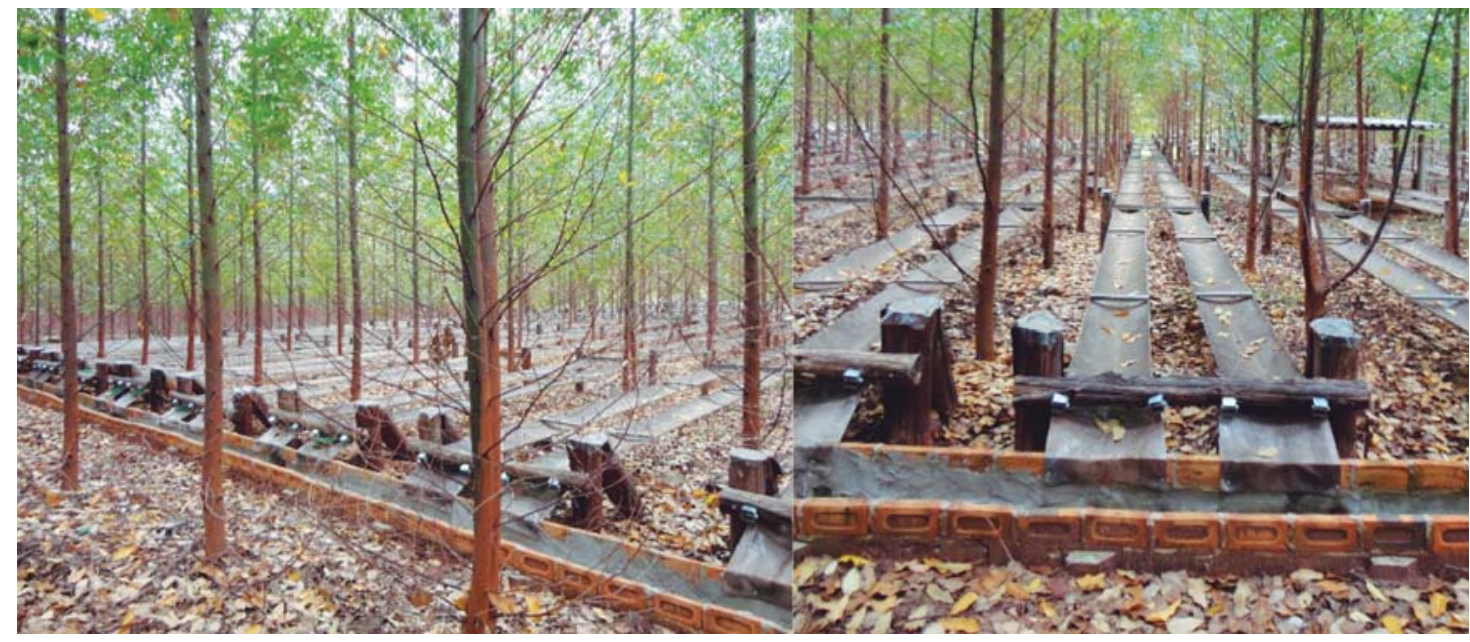

Figura 1 - Delineamento experimental com árvores de E. grandis. Detalhe da redução de chuva por faixas de polietileno. Figure 1 - Experimental design with E. grandis trees. Detail of rain reduction by polyethylene strips. 
A espessura da parede foi obtida pela razão da metade da diferença entre a largura e o diâmetro do lume. Para mensuração das fibras, foi utilizado o software Image Pro Plus, previamente calibrado para cada ampliação, e obtidos os valores de dimensões das fibras por tratamento.

Para avaliação dos vasos, foram utilizados os corpos de prova orientados, cortados nas três posições radiais, com dimensões de $20 \times 10 \mathrm{~mm}$ de largura e espessura, respectivamente; em seguida, foram imersas em água, em ebulição, para saturação, amolecimento e posterior fixação em micrótomo de deslize e corte das seções transversais, com espessura de 15 a $20 \mu \mathrm{m}$. Os cortes foram clarificados em água Cândida (1:1), lavados em água destilada em mistura de ácido acético $1 \%$, desidratados (série alcoólica, 30-100\%), lavados (xilol) e montados sob lamínula, com bálsamo-do-canadá. Das lâminas foram coletadas imagens do plano transversal (6 imagens por amostra), para a mensuração do diâmetro, porcentagem de área e frequência dos vasos $/ \mathrm{mm}^{2}$, aplicando-se o software Image Pro Plus e seguindo a Norma da IAWA (1989). As dimensões das fibras e dos vasos foram relacionadas com os tratamentos de nutrição x disponibilidade hídrica, posição radial e idade das árvores.

\subsection{Análise estatística}

$\mathrm{Na}$ análise estatística dos resultados, foi aplicado o programa JMP (SAS, 1997), sendo aferidos os outliers, a distribuição dos dados e da heterogeneidade da variância. Foram aplicadas duas análises de variância (ANOVA): uma, para verificar o efeito dos tratamentos: nutrição x disponibilidade hídrica e outra, para verificar o efeito dos fatores isolados: nutrição (controle, $\mathrm{Na}$ e K) e disponibilidade hídrica (66 e 100\% de chuva). Para tanto, foi aplicado o teste de Tukey, ajustado a $95 \%$ de probabilidade.

\section{RESULTADOS}

\subsection{Dimensões das fibras}

Foram observados efeitos significativos dos tratamentos de nutrição $\mathrm{x}$ disponibilidade hídrica nas dimensões das fibras no lenho de E. grandis aos 24 meses de idade (Tabela 1), exceto para o comprimento. Na idade de 36 meses, as dimensões das fibras no lenho das árvores não apresentaram diferenças significativas em razão dos tratamentos aplicados.
Os menores valores de largura das fibras foram observados no lenho das árvores submetidas aos tratamentos-controle e com adubação potássica e sódica, com redução de chuva $(17,39 ; 16,22$; e 17,36 $\mu \mathrm{m}$, respectivamente) aos 24 meses. O diâmetro do lume foi maior no lenho das árvores do tratamento com aplicação de $\mathrm{Na}$ e $100 \%$ de chuva $(12,36 \mu \mathrm{m})$, se comparado aos tratamentos $\mathrm{C} /-\mathrm{C}$ e Na/-C, com valores de 10,16 e 10,52 $\mu \mathrm{m}$, respectivamente. Com relação à espessura da parede, maiores valores foram encontrados no lenho das árvores submetidas aos tratamentos $\mathrm{C} /$ $+\mathrm{C}$ e $\mathrm{K} /+\mathrm{C}$, com 4,47 e 4,36 $\mu \mathrm{m}$, respectivamente.

Apesar do efeito não significativo, o valor médio do comprimento das fibras foi maior no lenho das árvores adubadas com $\mathrm{K}(817,62-832,46 \mu \mathrm{m}$ aos 24 meses e 908,70$937,71 \mu \mathrm{m}$ aos 36 meses), em relação aos tratamentos submetidos à nutrição-controle e $\mathrm{Na}$, nas condições com e sem redução de chuva (K/+C e K-C).

Avaliando o efeito isolado da nutrição em um regime hídrico natural (100\% de chuva; Tratamentos $\mathrm{C} /+\mathrm{C}$, $\mathrm{Na} /+\mathrm{C}$ e $\mathrm{K} /+\mathrm{C}$ ), verificou-se que a aplicação do $\mathrm{Na}$ propiciou o aumento do diâmetro do lume e a redução da espessura da parede, aos 24 meses (Tabela 1). Na idade de 36 meses, não foram observadas diferenças estatísticas significativas para as dimensões das fibras em função da aplicação de fertilizantes minerais.

$\mathrm{Na}$ avaliação isolada dos tratamentos de disponibilidade hídrica $(-\mathrm{C}$ e $+\mathrm{C})$, observou-se que a redução dos níveis de precipitação (Tratamentos C/ -C, $\mathrm{Na} /-\mathrm{C}$ e K/-C, considerando a média das duas idades) não influenciou significativamente $(\alpha=0,05)$ as características anatômicas do lenho, apesar de serem observadas ligeiras reduções das dimensões médias do comprimento $(827,76$ para $816,22 \mu \mathrm{m})$, da largura total $(21,12$ para $19,33 \mu \mathrm{m})$, do diâmetro do lume $(11,61$ para $11,09 \mu \mathrm{m})$ e da espessura da parede $(4,75$ para $4,11 \mu \mathrm{m})$, em relação aos valores médios encontrados no lenho das árvores submetidas aos tratamentos com $100 \%$ de chuva $(\mathrm{C} /+\mathrm{C}, \mathrm{Na} /+\mathrm{C}$ e $\mathrm{K} /+\mathrm{C}$, considerando a média das duas idades).

O efeito da idade das árvores nas dimensões médias das fibras foi significativo para a largura total e espessura da parede, sendo constatado o aumento dos valores de 24 (18,41 e 3,69, respectivamente) para 36 meses (22,03 e 5,17 $\mu \mathrm{m}$, respectivamente). Apesar de não significativo, o comprimento e o diâmetro das fibras também apresentaram tendências de aumento dos valores com o avanço da idade das árvores. 
Tabela 1 - Valores médios do comprimento, largura, espessura da parede e diâmetro do lume das fibras ( $\mu \mathrm{m})$ do lenho das árvores de E. grandis, com 24 e 36 meses, por tratamento de nutrição e disponibilidade hídrica.

Table 1 - Average length, width, wall thickness and lumen diameter of the fibers $(\mu \mathrm{m})$ of wood of $\mathbf{E}$. grandis trees, with 24 and 36 months, by fertilization treatment and water availability.

\begin{tabular}{|c|c|c|c|c|c|}
\hline $\begin{array}{c}\text { Idade } \\
\text { (meses) }\end{array}$ & Tratamentos & $\begin{array}{l}\text { Comprimento } \\
(\mu \mathrm{m})\end{array}$ & $\begin{array}{c}\text { Largura } \\
(\mu \mathrm{m})\end{array}$ & $\begin{array}{l}\text { Diâmetro do lume } \\
\qquad(\mu \mathrm{m})\end{array}$ & $\begin{array}{l}\text { Espessura da parede } \\
\qquad(\mu \mathrm{m})\end{array}$ \\
\hline \multirow[t]{6}{*}{24} & $\mathrm{C} /+\mathrm{C}$ & 762,93 a $(94,05)$ & 19,58 a $(4,49)$ & $10,62 \mathbf{a}$ & 4,47 a $(0,62)$ \\
\hline & $\mathrm{Na} /+\mathrm{C}$ & 774,05 a $(132,76)$ & 19,90 a $(4,17)$ & $12,36 \mathbf{b} \quad(2,89)$ & $3,76 \mathbf{b} \quad(0,47)$ \\
\hline & $\mathrm{K} /+\mathrm{C}$ & 832,46 a $(133,38)$ & 20,06 a $(4,20)$ & 11,33 ab $(2,94)$ & 4,36 a $(0,54)$ \\
\hline & $\mathrm{C} /-\mathrm{C}$ & 788,22 a $(131,55)$ & 17,39 b $(3,99)$ & 10,16 a $(2,47)$ & 3,61 bc $(0,45)$ \\
\hline & $\mathrm{Na} /-\mathrm{C}$ & 788,81 a $(112,76)$ & 16,22 b $(3,64)$ & 10,52 a $(2,05)$ & 2,85 d $\quad(0,31)$ \\
\hline & $\mathrm{K} /-\mathrm{C}$ & 817,62 a $(128,77)$ & 17,36 b $(4,43)$ & 11,15 ab $(2,49)$ & 3,10 cd $(0,32)$ \\
\hline Média & & $794,01 \mathbf{A}(118,16)$ & 18,41 A $(3,68)$ & 11,02 A $(2,01)$ & 3,69 A $(0,33)$ \\
\hline \multirow[t]{6}{*}{36} & $\mathrm{C} /+\mathrm{C}$ & 798,41 a $(139,68)$ & 22,56 a $(3,28)$ & 11,34 a $(2,56)$ & 5,61 a $(0,58)$ \\
\hline & $\mathrm{Na} /+\mathrm{C}$ & 859,02 a $(123,68)$ & 22,23 a $(3,24)$ & 12,09 a $(2,45)$ & 5,08 a $(0,45)$ \\
\hline & $\mathrm{K} /+\mathrm{C}$ & 937,71 a $(116,98)$ & 22,44 a $(4,25)$ & 11,96 a $(2,01)$ & 5,21 a $(0,52)$ \\
\hline & $\mathrm{C} /-\mathrm{C}$ & 803,56 a $(136,66)$ & 21,46 a $(3,61)$ & 11,22 a $(2,05)$ & 5,11 a $(0,67)$ \\
\hline & $\mathrm{Na} /-\mathrm{C}$ & 791,38 a $(125,29)$ & 22,25 a $(3,51)$ & 12,12 a $(2,01)$ & 4,96 a $(0,54)$ \\
\hline & $\mathrm{K} /-\mathrm{C}$ & 908,70 a $(138,19)$ & 21,34 a $(3,42)$ & $12,13 \mathbf{a}$ & 5,05 a \\
\hline Média & & 849,97 A $(159,44)$ & 22,03 B $(2,68)$ & 11,68 A $(1,98)$ & 5,17 B $\quad(0,63)$ \\
\hline
\end{tabular}

Médias seguidas de desvio-padrão. Médias seguidas da mesma letra, por idade e na coluna, não diferem entre si, a $95 \%$ de probabilidade, pelo teste de Tukey. Minúscula: comparação entre tratamentos e Maiúscula: comparação entre idade.

Means followed by standard deviation. Means followed by the same letter, per age and column, do not differ from each other at $95 \%$ probability by Tukey test. Small letter: comparison between treatments and Capital letter: comparison between ages.

As variações das dimensões das fibras nas três posições radiais $(0 \%, 50 \%$ e $100 \%)$ no DAP do tronco das árvores de eucalipto (Figura 2) indicam aumento significativo do comprimento $(620,88-722,95$ para 911,59 $1119,30 \mu \mathrm{m})$ da largura $(18,5-20,6$ para $19,51-22,28 \mu \mathrm{m})$, diâmetro do lume (10,06-11,64 para 11,12-13,09 $\mu \mathrm{m})$ e espessura da parede (3,93-4,83 para 3,96-5,30 $\mu \mathrm{m})$, comum em todos os tratamentos de nutrição $x$ disponibilidade hídrica aplicados.

\subsection{Dimensões e frequência dos vasos}

Para as dimensões e frequência dos vasos, não foram observados efeitos significativos dos tratamentos de nutrição x disponibilidade hídrica aos 24 e 36 meses de idade (Tabela 2).

Apesar do efeito não significativo, os valores médios do diâmetro dos vasos foi numericamente maior no lenho das árvores adubadas com K (90,46-107,87 $\mu \mathrm{m}$ aos 24 meses e 109,22-108,82 $\mu \mathrm{m}$ aos 36 meses) e Na (87,68-90,19 $\mu \mathrm{m}$ aos 24 meses e 94,47-97,87 $\mu \mathrm{m}$ aos 36 meses) em relação ao controle $(86,56-83,82 \mu \mathrm{m}$ aos 24 meses e 87,76-96,45 aos 36 meses) nos tratamentos com e sem redução de chuva. Entretanto, o número de vasos por unidade de área (frequência) foi numericamente menor no lenho das árvores adubadas

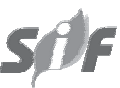

com potássio também nas duas condições de regime hídrico (100 e 66\% de chuva), aos 24 e 36 meses. Para a \% de área ocupada pelos vasos, não foi detectado padrão de variação em razão dos tratamentos aplicados.

Avaliando o efeito isolado da nutrição em regime hídrico natural (100\% de chuva; Tratamentos $\mathrm{C} /+\mathrm{C}$, $\mathrm{Na} /+\mathrm{C} \mathrm{e} \mathrm{K} /+\mathrm{C}$ ), verificou-se que não há efeito significativo da aplicação da nutrição mineral nas dimensões e frequência dos vasos, nas duas idades estudadas (Tabela 2). Apesar disso, a aplicação isolada de K promoveu um lenho com maior \% de área ocupada e diâmetro tangencial e menor frequência de vasos em relação ao controle e aplicação de $\mathrm{Na}$ aos 24 meses. Na idade de 36 meses, as tendências se mantêm, exceto para a \% de área ocupada, onde os maiores valores foram encontrados no lenho das árvores que receberam $\mathrm{Na}$.

Da mesma forma do que o observado nas dimensões das fibras, na avaliação isolada dos tratamentos de disponibilidade hídrica $(-\mathrm{C}$ e $+\mathrm{C})$ não foi verificado efeito significativo $(\alpha=0,05)$ da redução dos níveis de precipitação (Tratamentos C/-C, Na/-C e K/considerando a média das duas idades), apesar da redução dos valores do diâmetro tangencial $(97,45$ para $92,64 \mu \mathrm{m})$. A área ocupada e a frequência dos

Revista Árvore, Viçosa-MG, v.39, n.2, p.405-416, 2015 

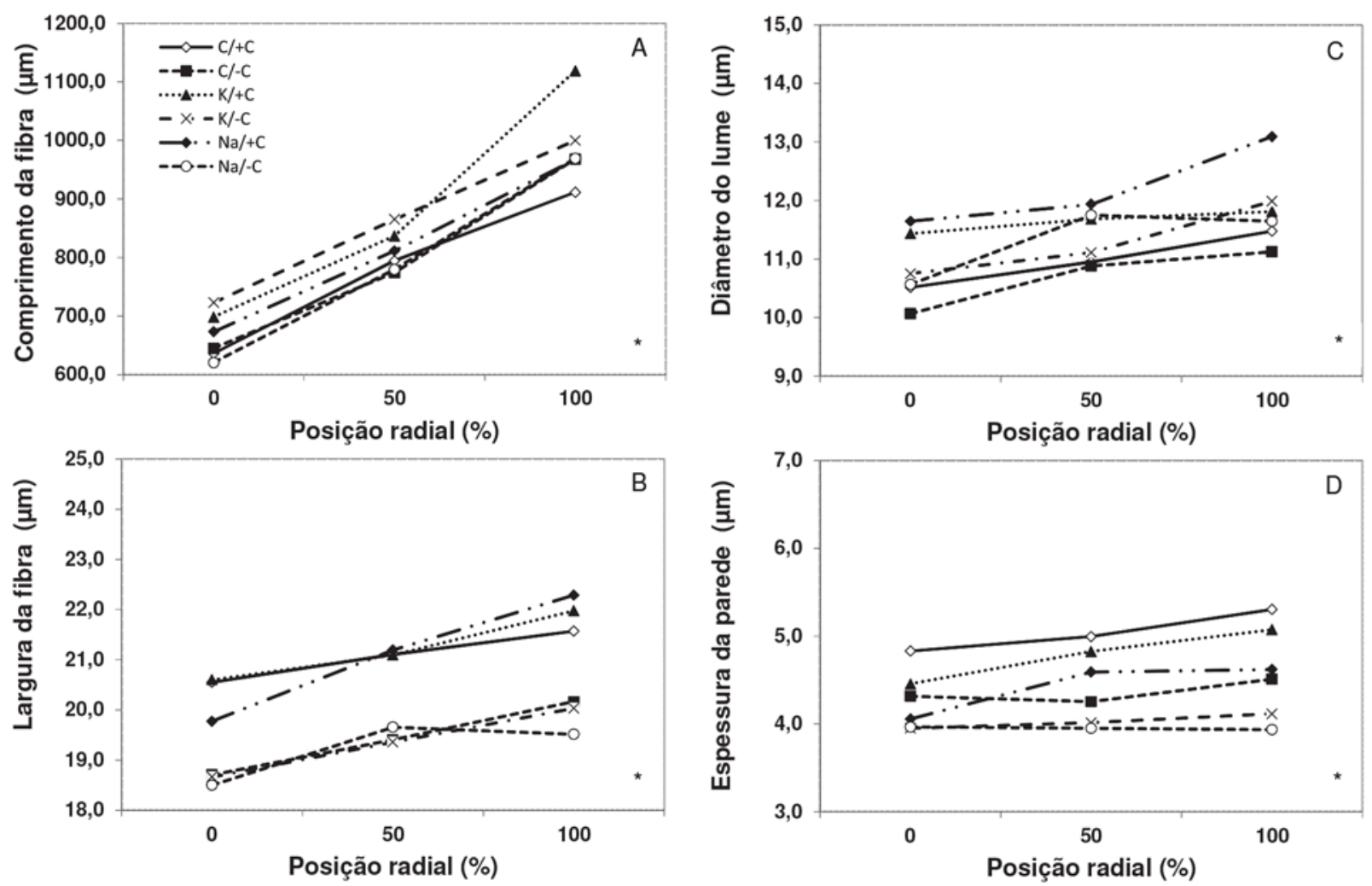

Figura 2 - Variação das dimensões médias das fibras no sentido radial; média das duas idades: comprimento (A), largura (B), diâmetro do lume (C) e espessura da parede (D), por tratamento de nutrição x disponibilidade hídrica. * Significativo $(\alpha=0,05)$.

Figure 2 - Variation of the mean fiber dimensions in the radial direction; average of two ages: length (A), width (B), lumen diameter $(C)$ and wall thickness $(D)$ per treatment of nutrition $x$ water availability. *Significant $(\alpha=0.05)$.

vasos não se alteraram nas duas condições hídricas, como valores médios de 20 vasos $/ \mathrm{mm}^{2}$ e $14 \%$, respectivamente.

A porcentagem de área ocupada aumentou significativamente de 24 para 36 meses, passando de 13 para $15 \%$. Para as demais variáveis dos elementos de vaso, apesar de não significativo, observou-se tendência de aumento da frequência (19 para 20\%) e diâmetro tangencial (91 para $92 \mu \mathrm{m}$ ), com o incremento da idade das árvores de E. grandis.

Nos três parâmetros analisados dos vasos, foi detectado efeito significativo da posição radial, sendo observado aumento no diâmetro tangencial (74,2$81,6$ para $100,0-127,1 \mathrm{ìm})$ e na área ocupada (11-13 para $13-17 \%)$ e redução na frequência (22-25 para 12-19 vasos. $\mathrm{mm}^{-2}$ ) dos vasos da medula para a casca (Figura 3).

\section{DISCUSSÃO}

Os valores médios das dimensões das fibras do lenho de $E$. grandis e da sua relação com os tratamentos de nutrição $\mathrm{x}$ disponibilidade hídrica obtidos neste estudo indicaram efeitos significativos aos 24 meses, exceto para o comprimento das fibras e não significativos aos 36 meses (Tabela 1). Para as dimensões e frequência dos vasos, não foram notados efeitos significativos dos tratamentos aplicados aos 24 e 36 meses de idade (Tabela 2).

A literatura especializada possui poucas e controversas informações a respeito do efeito da disponibilidade hídrica (irrigação) e da aplicação de fertilizantes associados à irrigação nas dimensões das fibras e vasos no lenho de árvores de eucalipto. Os resultados de pesquisas realizadas por Beadle et al. (2001), Clark et al. (2004) e Tomazello Filho (2006) indicaram

Revista Árvore, Viçosa-MG, v.39, n.2, p.405-416, 2015 
Tabela 2 - Valores médios da \% de área ocupada, frequência $\left(\mathrm{n}^{\%} / \mathrm{mm}^{2}\right)$ e diâmetro tangencial $(\mu \mathrm{m})$ dos vasos do lenho das árvores de E. grandis, com 24 e 36 meses, por tratamento de nutrição e disponibilidade hídrica.

Table 2 - Mean values of \% of occupied area, frequency $\left(\mathrm{n}^{\circ} / \mathrm{mm}^{2}\right)$ and tangential diameter $(\mu \mathrm{m})$ of the vessels of the wood of $\mathbf{E}$. grandis trees, with 24 and 36 months, by fertilization treatment and water availability.

\begin{tabular}{|c|c|c|c|c|}
\hline $\begin{array}{l}\text { Idade } \\
(\text { meses })\end{array}$ & Tratamentos & \% área ocupada & Frequência $\left(\mathrm{n}^{\circ} / \mathrm{mm}^{2}\right)$ & Diâmetro tangencial $(\mu \mathrm{m})$ \\
\hline \multirow[t]{6}{*}{24} & $\mathrm{C} /+\mathrm{C}$ & 12,50 a $(1,40)$ & 18,50 a $(2,54)$ & 83,82 a $(12,65)$ \\
\hline & $\mathrm{Na} /+\mathrm{C}$ & 12,64 a $(1,56)$ & 18,52 a $(2,10)$ & 90,19 a $(13,66)$ \\
\hline & $\mathrm{K} /+\mathrm{C}$ & 14,62 a $(2,72)$ & 15,87 a $(2,10)$ & 107,87 a $(17,43)$ \\
\hline & $\mathrm{C} /-\mathrm{C}$ & 12,60 a $(2,35)$ & 23,97 a $(1,48)$ & 86,26 a $(13,06)$ \\
\hline & $\mathrm{Na} /-\mathrm{C}$ & 12,15 a $(1,23)$ & 19,61 a $(2,04)$ & 87,68 a $(12,86)$ \\
\hline & $\mathrm{K} /-\mathrm{C}$ & 12,48 a $(2,65)$ & 16,87 a $(2,00)$ & 90,46 a $(13,81)$ \\
\hline Média & & 12,86 A $(1,93)$ & 18,89 A $(3,20)$ & $91,07 \mathbf{A}(14,20)$ \\
\hline \multirow[t]{6}{*}{36} & $\mathrm{C} /+\mathrm{C}$ & 13,71 a $(1,88)$ & 23,32 a $(2,17)$ & 96,45 a $(14,96)$ \\
\hline & $\mathrm{Na} /+\mathrm{C}$ & 18,48 a $(3,48)$ & 19,50 a $(2,66)$ & 97,87 a $(13,70)$ \\
\hline & $\mathrm{K} /+\mathrm{C}$ & 14,95 a $(2,84)$ & 16,45 a $(2,77)$ & 108,82 a $(17,67)$ \\
\hline & $\mathrm{C} /-\mathrm{C}$ & 15,20 a $(2,81)$ & 20,85 a $(2,52)$ & 87,76 a $(12,34)$ \\
\hline & $\mathrm{Na} /-\mathrm{C}$ & 14,22 a $(4,05)$ & 19,57 a $(2,46)$ & 94,47 a $(15,38)$ \\
\hline & $\mathrm{K} /-\mathrm{C}$ & 16,32 a $(3,60)$ & 19,24 a $(2,81)$ & 109,22 a $(18,15)$ \\
\hline Média & & 15,48 B $(3,14)$ & 19,82 A $(2,91)$ & 98,89 A $(15,31)$ \\
\hline
\end{tabular}

Médias seguidas de desvio-padrão. Médias seguidas da mesma letra, por idade e na coluna, não diferem entre si, a 95\% de probabilidade, pelo teste de Tukey. Minúscula: comparação entre tratamentos e Maiúscula: comparação entre idade.

Means followed by standard deviation. Means followed by the same letter, per age and column, do not differ from each other at $95 \%$ probability by Tukey test. Small letter: comparison between treatments and Capital letter: comparison between ages.

que maior disponibilidade hídrica induz a formação de fibras mais longas no lenho de árvores de Eucalyptus spp. Entretanto, Castro (2014) e Pereira et al. (1994) não detectaram a influência da disponibilidade hídrica no comprimento das fibras no lenho de diversas espécies de eucalipto, e Tomazello Filho (2006) não observou diferenças significativas nas dimensões dos vasos no lenho de árvores de E. grandis x urophylla aos 7 anos, submetidas a tratamentos de nutrição e irrigação.

Com relação aos valores médios de largura de fibra no lenho das árvores dos seis tratamentos de nutrição $\mathrm{x}$ disponibilidade hídrica (Tabela 1), alguns autores como Larson (1973) e Shimoyamae Barrichello (1989) mencionaram tais diferenças em fatores genéticos e ambientais, além do crescimento sazonal. Na primavera e no verão, por exemplo, a árvore produz maior quantidade de hormônios, acarretando maiores aumentos na largura das células. Hormônios específicos (auxina, por exemplo) agem no arranjo das fibrilas, ora afrouxando-as, por meio de aumento do ângulo fibrilar e aumento do diâmetro da fibra, ora concentrando-as, pela diminuição do ângulo fibrilar, acarretando diminuição na largura das fibras.

Com relação à espessura de parede das fibras no DAP do lenho das árvores nos seis tratamentos, Shimoyama e Barrichello (1989) afirmaram que o espessamento da parede está relacionado a fatores genéticos e ambientais, bem como a idade da árvore. Relacionando o estado nutricional e a disponibilidade hídrica, as árvores sintetizam maior quantidade de açúcares simples pela fotossíntese e, na camada cambial, propiciam aumento da biossíntese de moléculas de celulose que, em seguida, são incorporadas como microfibrilas, resultando no espessamento da parede secundária. A literatura especializada relata que o espessamento da parede celular - representado pela formação da parede secundária, pela deposição de microfibrilas - relaciona-se com os níveis de carboidratos elaborados no processo de fotossíntese (LARSON, 1973). Segundo Panshin e De Zeeuw (1970), o espessamento ocorre com a deposição de carboidratos, principalmente quando diminui o crescimento da árvore.

A redução dos níveis de precipitação (Tratamentos $\mathrm{C} /-\mathrm{C}, \mathrm{Na} /-\mathrm{C}$ e K/-C, considerando a média das duas idades) não influenciou significativamente $(a ́=0,05)$ as características anatômicas do lenho, embora tenham sido constatadas tendências de redução nos valores com o estresse hídrico. Diferentemente do observado neste trabalho, pesquisas recentes realizadas por Drew et al. (2009) e Sette JR. et al. (2013) demonstraram alta sensibilidade das fibras e dos vasos aos níveis de

Revista Árvore, Viçosa-MG, v.39, n.2, p.405-416, 2015 

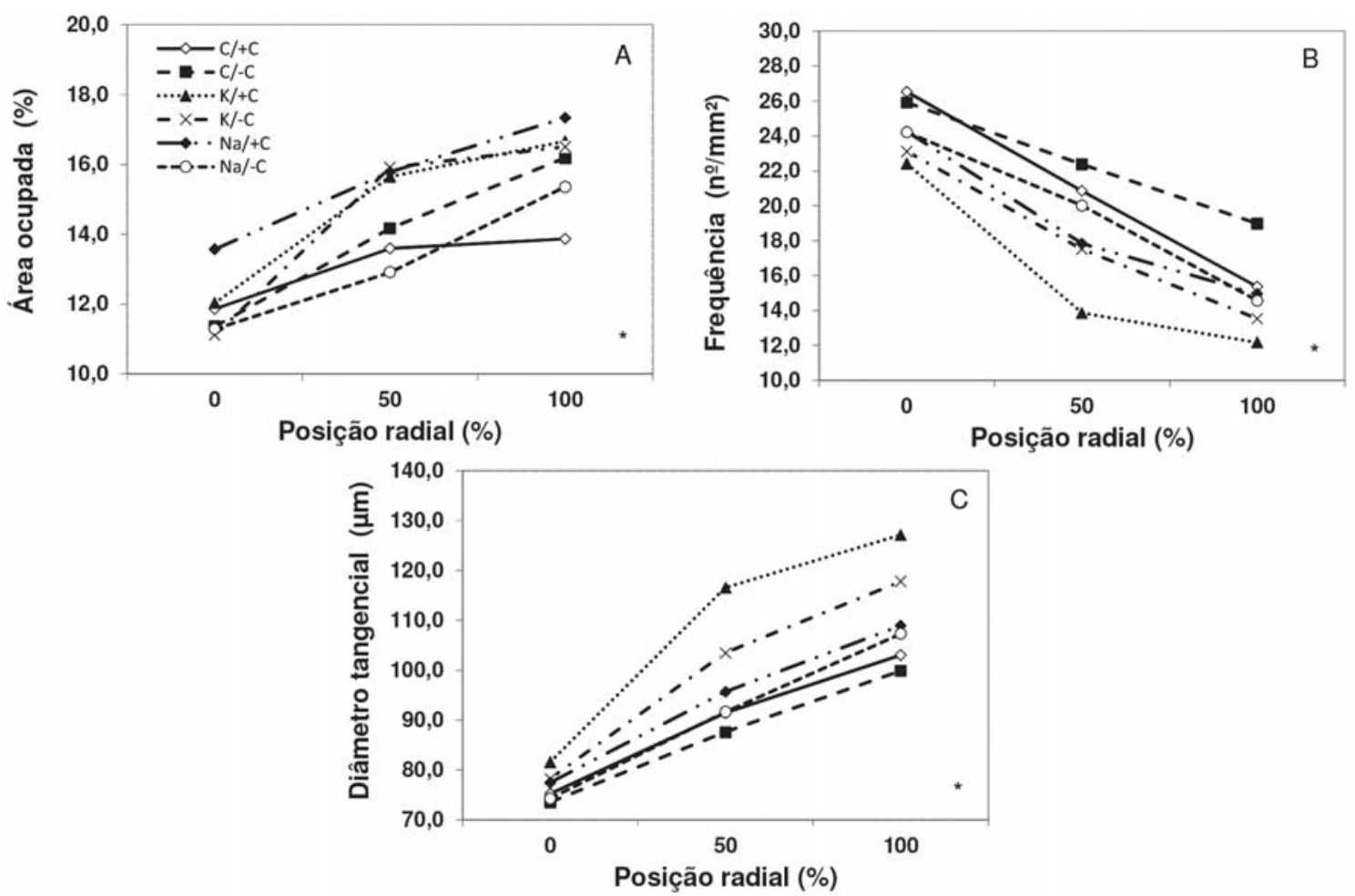

Figura 3 - Variação das dimensões dos vasos no sentido radial; média das duas idades: \% de área ocupada (A), frequência (B) e diâmetro tangencial $(C)$, por tratamento de nutrição $x$ disponibilidade hídrica. $*$ Significativo $(\alpha=0,05)$.

Figure 3 - Variation of the vessels dimensions in the radial direction; average of two ages: \% of area occupied area (A), frequency $(B)$ and tangential diameter $(C)$ per treatment of nutrition $x$ water availability. *Significant $(\alpha=0.05)$.

precipitação, sendo, contudo, observadas normalmente alterações significativas nas suas dimensões, com baixos níveis de chuva em função da redução da taxa de atividade cambial e, consequentemente, do crescimento em diâmetro do tronco das árvores.

Diferentemente do esperado, os resultados da avaliação da taxa de crescimento em diâmetro do tronco realizada com dendrômetros de aço instalados no DAP das árvores de eucalipto do mesmo experimento, apresentados por Castro (2014), demonstram que os tratamentos de redução de chuva apresentaram crescimentos superiores, em determinadas épocas do ano, até a idade de 24 meses, comparados aos tratamentos sem redução de chuva. Isso pode explicar os comportamentos não esperados das dimensões das características anatômicas do lenho.

Possivelmente, tal situação se deve às seguintes hipóteses: (i) nos dois anos iniciais, as lonas de plástico propiciaram aumento da temperatura, propiciando microclima favorável ao crescimento das árvores, com redução de chuva - hipótese comprovada por Cheesman e Winter (2013); (ii) nos primeiros anos, havia água depositada nas camadas profundas do solo; (iii) desenvolvimento de raízes finas nas camadas mais profundas do solo, estimuladas pela redução de chuva, como resposta adaptativa ao estresse hídrico, uma vez que as raízes finas são mais sensíveis à menor quantidade de chuvas no período mais seco, absorvendo a água disponível, de forma mais rápida e eficiente - hipótese comprovada por Laclau et al. (2013); (iv) a porcentagem de redução de chuva provavelmente não foi suficiente para promover forte estresse hídrico que afetasse o crescimento das plantas.

De acordo com Battie-Laclau et al. (2013, 2014), utilizando a mesma área experimental deste estudo, essas hipóteses são fundamentadas por variáveis que afetam o crescimento e desenvolvimento das árvores de eucalipto, como a sua área e queda foliar (tempo

Revista Árvore, Viçosa-MG, v.39, n.2, p.405-416, 2015 
de vida das folhas), atividade fotossintética, densidade e área dos estômatos (trocas gasosas), alocação do carbono nas partes da planta, potencial de base e osmótico, déficit de pressão de vapor e porcentagem de umidade ao longo da profundidade do solo, temperatura mínima do ar, entre outros.

Para poder explicar melhor o comportamento das árvores de eucalipto submetidas a tratamentos de redução artificial de chuva, é preciso avaliar o experimento em idades mais avançadas, construindo uma base de dados e uma série temporal que permita tirar conclusões e avaliar os efeitos da disponibilidade hídrica no funcionamento das plantações florestais de eucalipto, incluindo na qualidade da madeira. Cabe ressaltar ainda que avaliações de outros parâmetros, como área foliar, queda foliar (tempo de vida das folhas), atividade foliar fotossintética, densidade e área ocupada por estômatos, alocação de carbono em diferentes partes da planta, potencial osmótico, déficit de pressão de vapor e teor de umidade em diferentes profundidades do solo influenciam essa análise e devem ser considerados nas avaliações.

A aplicação do K nas árvores submetidas à redução de chuva (Tratamento K/-C) não influenciou diretamente as dimensões das fibras aos 36 meses e dos vasos aos 24 e 36 meses, apresentando valores iguais estatisticamente ao daquelas árvores que receberam o nutriente, porém não foram submetidas à redução de chuva (Tabelas 1 e 2). Esse resultado indica, até o momento (36 meses), que a aplicação de K possivelmente não aumentou a eficiência do uso da água pelas plantas sob estresse hídrico, conforme sugere alguns trabalhos (GONÇALVES et al., 2004; ALMEIDA, 2009), não aumentando a resistência das árvores à seca e, consequentemente, não alterando as características anatômicas do lenho. De acordo com Sette JR. et al. (2009), a avaliação das dimensões das fibras e dos elementos de vaso e a formação do cerne e do alburno nas árvores de eucalipto, em idades mais avançadas, são fundamentais para se entender o mecanismo de fluxo ascendente de seiva bruta no xilema funcional do tronco das árvores de diferentes volumes de lenho e de área foliar, submetidas aos diferentes tratamentos de nutrição e disponibilidade hídrica.

A avaliação isolada do efeito da aplicação do K e do $\mathrm{Na}$ indicou não influenciar as características anatômicas do lenho do E. grandis, exceto para a largura, diâmetro do lume e espessura da parede aos 24 meses
(Tabelas 2 e 3). Em árvores de espécies de eucalipto de idades avançadas (até 30 anos), os resultados de pesquisas referentes ao efeito isolado de fertilizantes minerais nas propriedades da madeira são controversos, com indicação, por exemplo, de aumento (VIGNERON et al., 1995) e redução (WASHUSEN et al., 2005) da densidade do lenho. Em árvores de eucalipto jovens (1-4 anos), destacam-se os resultados de Bamber et al. (1982), Pereira e Araujo (1990) e Sette JR. et al. (2009), que não verificaram efeito significativo da nutrição mineral nas dimensões das fibras e dos vasos.

Para as variações das dimensões médias das fibras e dos vasos, nas duas idades, observaram-se tendências de aumento dos valores com o avanço da idade das árvores, nem sempre significativo (Tabelas 1 e 2). Esse resultado corrobora os obtidos por Sette JR. et al. (2014, 2012) e Trugilho (1996), ao estudarem a influência da idade nas dimensões das fibras e dos vasos do eucalipto. O aumento do comprimento das fibras em razão da idade é resultado do aumento do comprimento das células que as originam, denominadas iniciais fusiformes. A estabilização do comprimento das fibras, em inúmeras espécies de eucalipto, somente irá ocorrer quando as células do câmbio atingirem comprimento máximo, iniciando a formação do lenho adulto (TRUGILHO, 1996; SETTE JR. et al., 2012).

As variações das dimensões das fibras e dos vasos nas três posições radiais $(0 \%, 50 \%$ e $100 \%)$ no DAP do tronco das árvores de eucalipto (Figuras 2 e 3 ) indicaram modelos de variação comum a todos os tratamentos e características da espécie. A literatura científica reporta extensa lista de artigos, relatando a variação das dimensões das fibras e dos vasos, no sentido radial do lenho de árvores de diferentes espécies de eucalipto e condições de crescimento, indicando, entre outros, os observados neste estudo (SETTE JR. et al., 2009, 2012).

\section{CONCLUSÃO}

Os tratamentos de nutrição $\mathrm{x}$ disponibilidade hídrica induziram alterações na largura, diâmetro do lume e espessura da parede da fibra aos 24 meses e não alteraram as dimensões dos vasos no lenho do E. grandis. A aplicação isolada do K e do Na não influenciou as características anatômicas do lenho do E. grandis, exceto na largura, diâmetro do lume e espessura da parede aos 24 meses. A redução dos níveis de precipitação, pela instalação de faixas de

Revista Árvore, Viçosa-MG, v.39, n.2, p.405-416, 2015

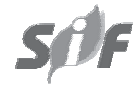


lonas de polietileno, não influenciou significativamente as dimensões das fibras e dos vasos no lenho do E. grandis.

Os resultados poderão ser utilizados como subsídios para a adoção de práticas silviculturais nas plantações florestais em áreas com estresse hídrico, bem como a substituição parcial de $\mathrm{K}$ por $\mathrm{Na}$.

\section{AGRADECIMENTOS}

Esta pesquisa foi parcialmente financiada pelo projeto apoiado pela Fundação Agropolis sob o ID de referência 1203-003, através do "Investissements d'avenir programme" (Labex Agro:ANR-10-LABX0001-01).

\section{REFERÊNCIAS}

ALMEIDA, J. C. R. Nutrição, crescimento, eficiência de uso da água e de nutrientes em povoamentos de Eucalyptus grandis fertilizados com potássio e sódio. 2009. 112f. Tese (Doutorado em Recursos Florestais) - Escola Superior de Agricultura “Luiz de Queiroz”. Piracicaba, 2009.

BAMBER, R.K.; HORNE, R.; GRAHAM-HIGGS, A. Effect of fast growth on the wood properties of Eucalyptus grandis. Australian Forestry Research, v.12, n.2, p.163-167, 1982.

BATTIE-LACLAU, P.; LACLAU, J.P.; PICCOLO, M.C.P. Influence of potassium and sodium nutrition on leaf area components in Eucalyptus grandis trees. Plant and Soil, v.371, n.1, p.19$35,2013$.

BATTIE-LACLAU, P. et al. Photosynthetic and anatomical responses of Eucalyptus grandis leaves to potassium and sodium supply in a field experiment. Plant, Cell \& Environment, v.37, n.1, p.70-81, 2014.

BEADLE, C.; BANHAM, P. W.; WORLEDGE, D.; RUSSEL, S. L.; HETERINGTON, S. J.; HONEYSETT, J. L.; WHITE, D. Effect of irrigation on growth and fibre quality of Eucalytpus globulus and Eucalyptus nitens. Appita Journal, v.54, n.2, p.144-147, 2001.

CABRAL, O. M. R.; ROCHA, H. R.; GASH, J. H. C.; LIGO, M. A. V.; FREITAS, H. C.; TATSCH, J. D. The energy and water balance of a Eucalyptus plantation in southeast Brazil. Journal of Hydrology, v.388, p.208-216, 2010.

CASTRO, V.R. Efeitos do potássio, sódio e da disponibilidade hídrica no crescimento e qualidade do lenho de árvores de Eucalyptus grandis Hill ex Maiden. 2014. 177f. Tese (Doutorado em Ciências Florestais) - Escola Superior de Agricultura "Luiz de Queiroz", Piracicaba, 2014.

CHEESMAN, A.W.; WINTER, K. Elevated nighttime temperatures increase growth in seedlings of two tropical pioneer tree species. New

Phytologist, v.197, n.4, p.1185-1192, 2013.

CLARK, D.A. Sources or sinks? The responses of tropical forests to current and future climate and atmospheric composition. Philosophical Transactions of the Royal Society, v.359, n.1443, p.477-491, 2004.

COOPMAN, R. E.; JARA, J. C.; BRAVO, L. A.; SÁEZ, K. L.; MELLA, G. R.; ESCOBAR, R. Changes in morpho-physiological attributes of Eucalyptus globulus plants in response to different drought hardening treatments.

Electronic Journal of Biotechnology, v.11, n.2, p.30-39, 2008.

DREW, D. M.; GEOFFRE, Y. M.; DOWNES, G. M.; GRADY, A. P.; READ, J.; WORLEDGE, D. High resolution temporal variation in wood properties in irrigated and non-irrigated Eucalyptus globulus. Annals of Forest Science, v.66, p.1-10, 2009.

GAVA, J. L. Efeito de adubação potássica em plantios de E. grandis conduzidos de segunda rotação em solos com diferentes teores de potássio trocável. Série Técnica IPEF, v. 11, p. 89-94, 1997.

FROMM, J. Wood formation of trees in relation to potassium and calcium nutrition. Tree Physiology, v.30, n.9, p.1140-1147, 2010.

GONÇALVES, J. L. M.; BENEDETTI, V. Nutrição e fertilização florestal. Piracicaba: IPEF, 2000. 427p.

GONÇALVES, J. L. M.; STAPE, J. L.; BENEDETTI, V.; FESSEL, V. A. G.; GAVA, J. L. An evaluation of minimum and intensive soil preparation regarding 
fertility and tree nutrition. In: GONÇALVES, J.L.M.; BENEDETII, V., (Ed.) Forest nutrition and fertilization. Piracicaba: IPEF, 2004. p.1364.

IAWA COMMITTEE. IAWA list of microscopic features for hardwood identification. In: WHEELER, E.A.; BAAS, P.; GASSON, P.E. (Ed.). IAWA Bulletin v.10, n.3, p.219-332, 1989.

INTERGOVERNMENTAL PANEL ON CLIMATE CHANGE - IPCC. Climate change 2007 The Physical Science Basis: contribution of Working Group I to the Fourth Assessment Report of the IPCC. Cambridge: Cambridge University Press, 2007. 996p.

JOHANSEN, D.A. Plant microtechnique. New York: MacGraw-Hill, 1940. 533p.

LACLAU, J.P.; SILVA, E.A.; LAMBAIS, G. Dynamics of soil exploration by fine roots down to a depth of $10 \mathrm{~m}$ in Eucalyptus grandis plantations. Frontiers in Plant Science, v.4, n.1, p.1-12, 2013.

LARSON, P. R. The physiological basis for wood specific gravity in conifers. IUFRO V Proceedings South Africa. Brisbane: 1973. p.672-680.

NOBRE, C.A.; SALAZAR, L. F.; OYAMA, M.E. Mudanças climáticas e possíveis alterações nos biomas da América do Sul. Brasília: Ministério do Meio Ambiente, 2007. 29p. (Relatório, 6)

PANSHIN, A.J.; DE ZEEUUW, C. Textbook of technology. 3.ed. New York, McGraw Hill, 1970. $705 \mathrm{p}$.

PEREIRA, H.; ARAUJO, C. Raw-material quality of fast grow Eucalyptus globulus during the first year. IAWA Bulletin, v.11, n.4, p.421-427, 1990.

PEREIRA, J. S.; MADEIRA, M.; LINDER. S; ERICSSON, T.; TOMÉ, M.; ARAÚJO, M. C. Biomass production with optimized nutrition in Eucalyptus globulus plantations. In: PERIERA, J.S.; PEREIRA, H. (Ed.), Eucalyptus for biomass production. Brussells: Commission of the European Communities, 1994. p.13-30.
SAS Institute. SAS/STAT User's Guide: vertion 6.08. Cary: 1997. v.2.846p.

SETTE JR., C. R. ; TOMAZELLO FILHO, M. ; DIAS, C. T. S. ; CHAGAS, M. P. ; LACLAU, J. P. Efeito da aplicação de potássio e sódio nas características do lenho de árvores de Eucalyptus grandis W Hill, aos 24 meses de idade. Revista Floresta, v.39, n.3, p.535-546, 2009.

SETTE JR., C. R.; TOMAZELLO FILHO, M. ; LOUSADA, J. L. P. C. ; LACLAU, J. P. Crescimento em diâmetro do tronco das árvores de Eucalyptus grandis e relação com as variáveis climáticas e fertilização mineral. Revista Árvore, v.34, p. 979-990, 2010.

SETTE JR., C. R.; ROSADA, I.; TOMAZELLO FILHO, M.; YAMAJI, F. M.; LACLAU, J. P. Efeito da idade e posição de amostragem na densidade e características anatômicas da madeira de Eucalyptus grandis. Revista Árvore, v.36, n.6, p.1183-1190, 2012.

SETTE JR., C. R.; LACLAU, J. P.; TOMAZELLO FILHO, M.; MOREIRA, R. M.; BOUILLET, J-P.; RANGER, J; ALMEIDA, J. C. R. Source-driven remobilizations of nutrients within stem wood in Eucalyptus grandis plantations. Trees -

Structure and Function, v.27, n.4, p.827839, 2013.

SETTE JR., C. R.; TOMAZELLO FILHO, M.; SILVA, F. G. ; LACLAU, J. P. Alterações nas características químicas da madeira com a substituição do K por $\mathrm{Na}$ em plantações de eucalipto. Revista Árvore, v.38, n.3, p.569$578,2014$.

SHIMOYAMA, V. R. D. S.; BARRICHELO, L. E. G. Importância da adubação na qualidade da madeira e celulose. Simpósio sobre adubação e qualidade dos produtos agrícolas. Ilha Solteira: 1989. p.1-15.

TOMAZELLO FILHO, M. Efeito da irrigação e fertilização nas propriedades doo lenho de árvores de Eucalyptus grandis $x$ urophila. Piracicaba: Escola Superior de Agricultura "Luiz de Queiroz", Universidade de São Paulo, 2006.

Revista Árvore, Viçosa-MG, v.39, n.2, p.405-416, 2015 
TRUGILHO, P.F. Influência da idade nas características físico-químicas e anatômicas da madeira de Eucalyptus saligna, Cerne, v.2, n.1, p.94-111, 1996.

VIGNERON, J.; GERARD, J.; BOUVET, J.M.

Relationship between basic density and growth in a fertilization trial with clones of Eucalyptus hybrids in Congo. In: IUFRO CONFERENCE EUCALYPT PLANTATIONS: IMPROVING FIBRE YIELD AND QUALITY, 1995, Hobart.
Proceedings... Hobart: IUFRO, 1995. p.68-71.

WASHUSEN, R.; BAKER, T.; MENZ, D.;

MORROW, A. Effect of thinning and fertilizer on the cellulose crystallite width of Eucalyptus globulus. Wood Science and Technology, v.39, n.7, p.569-578, 2005.

WIND, C.; AREND, M.; FROMM, J. Potassiumdependent cambial growth in poplar. Plant Biology, v.6, n.1, p.30-37, 2004. 